\title{
Characteristics and outcomes of public bath-related out-of-hospital cardiac arrests in South Korea
}

\author{
Yung Jae Yoo', Gi Woon Kim', Choung Ah Lee'2, Yong Jin Park', \\ Kyoung Mi Lee ${ }^{4}$, Jin Seong $\mathrm{Cho}^{5}$, Won Jung Jeong ${ }^{6}$, Hyuk Joong Choi ${ }^{7}$, \\ Han Joo Choi ${ }^{8}$, Nam Hun $\mathrm{Heo}^{9}$, Hyung Jun Moon ${ }^{1}$
}

\author{
1 Department of Emergency Medicine, Soonchunhyang University College of Medicine, Asan, Korea \\ ${ }^{2}$ Department of Emergency Medicine, Hallym University Dongtan Sacred Heart Hospital, Dongtan, Korea \\ ${ }^{3}$ Department of Emergency Medicine, Chosun University Hospital, Gwangju, Korea \\ ${ }^{4}$ Department of Emergency Medicine, Myongji Hospital, Goyang, Korea \\ ${ }^{5}$ Department of Emergency Medicine, Gachon University Gil Medical Center, Incheon, Korea \\ ${ }^{6}$ Department of Emergency Medicine, St. Vincent's Hospital, The Catholic University of Korea, Suwon, Korea \\ ${ }^{7}$ Department of Emergency Medicine, Hanyang University Guri Hospital, Guri, Korea \\ ${ }^{8}$ Department of Emergency Medicine, Dankook University Hospital, Cheonan, Korea \\ ${ }^{9}$ Clinical Trial Center, Soonchunhyang University Cheonan Hospital, Cheonan, Korea
}

Objective To analyze the differences in characteristics and outcomes between public bath (PB)related and non-PB-related out-of-hospital cardiac arrest (OHCA) patients in South Korea.

Methods We performed a retrospective observational analysis of collected data from the Smart Advanced Cardiac Life Support (SALS) registry between September 2015 and December 2018. We included adult OHCA patients (aged $>18$ years) with presumed OHCA of non-traumatic etiology who were attended by dispatched emergency medical services. SALS is a field advanced life support with smartphone-based direct medical direction. The primary outcome was the survival to discharge rate measured at the time of discharge.

Results Of 38,995 cardiac arrest patients enrolled in the SALS registry, 11,889 were included in the final analysis. In total, $263 \mathrm{OHCAs}$ occurred in PBs. Male sex and bystander cardiopulmonary resuscitation proportions appeared to be higher among PB patients than among non-PB patients. Percentages for shockable rhythm, witnessed rate, and number of underlying disease were lower in the PB group than in the non-PB group. Prehospital return of spontaneous circulation (11.4\% vs. $19.5 \%, P=0.001)$, survival to discharge $(2.3 \%$ vs. $9.9 \%, P<0.001)$, and favorable neurologic outcome $(1.9 \%$ vs. $5.8 \%, P=0.007)$ in $P B$ patients were significantly poorer than those in non-PB patients.

Conclusion Patient characteristics and emergency medical services factors differed between PB and non-PB patients. All outcomes of PB-related OHCA were poorer than those of non-PB-related OHCA. Further treatment strategies should be developed to improve the outcomes of PBrelated cardiac arrest.

Keywords Out-of-hospital cardiac arrest; Emergency medical services; Resuscitation; Baths
elSSN: 2383-4625

Received: 26 August 2019

Revised: 30 September 2019

Accepted: 1 October 2019

Correspondence to: Hyung Jun Moon Department of Emergency Medicine, Soonchunhyang University College of Medicine, 22 Soonchunhyang-ro, Asan 31538, Korea

E-mail: raintree@schmc.ac.kr ORCID

https://orcid.org/0000-0001-9985-8100

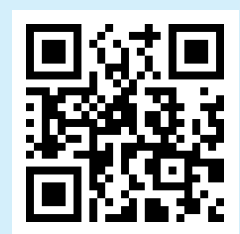

How to cite this article:

Yoo YJ, Kim GW, Lee CA, Park YJ, Lee KM, Cho JS, Jeong WJ, Choi HJ, Choi HJ, $\mathrm{Heo} \mathrm{NH}$, Moon HJ. Characteristics and outcomes of public bath-related out-ofhospital cardiac arrest in South Korea. Clin Exp Emerg Med 2020;7(3):225-233. https://doi. org/10.15441/ceem.19.071
This is an Open Access article distributed under the terms of the Creative Commons Attribution Non-Commercial License (https:// creativecommons.org/licenses/by-nc/4.0/). 


Capsule $\begin{aligned} & \text { What is already known } \\ & \text { It is estimated that the outcome of public bath-related cardiac arrest patients is poor. However, the majority of research } \\ & \text { examining this bath-related cardiac arrest has been conducted in Japan. }\end{aligned}$
$\begin{aligned} & \text { What is new in the current study } \\ & \text { We found that the characteristics of public bath-related out-of-hospital-cardiac arrest (OHCA) patients were different } \\ & \text { from others. Overall outcomes of public bath-related OHCAs were significantly worse than other OHCA patients. Other } \\ & \text { strategies should be provided to public bath OHCA patients in addition to advanced life support. }\end{aligned}$

\section{INTRODUCTION}

Sauna bathing is a passive heat therapy that is mainly used for the purposes of relaxation, wellness, and pleasure.' Sauna bathing is associated with several health benefits such as reduced risks of adverse cardiovascular and non-cardiovascular disease outcomes, as well as mortality. ${ }^{2}$ All plausible pathways (reduced arterial stiffness, decreases in inflammation and oxidative stress, stabilization of the autonomic nervous system, beneficial changes in circulating lipid profiles, and lowering of systemic blood pressure) contribute to the beneficial cardiovascular health effects and may explain the protective effect of sauna bathing on sudden cardiac arrest. Most studies on bath-related cardiac arrest were conducted in Japan. ${ }^{3-10}$ Japanese have the habit of almost daily, long, head-out-of-water immersion style sitting in hot water bathtubs. ${ }^{11}$ In Japan, cardiac arrest occurring in the bath typically occurs in people older than 70 years during the winter. Satoh et al. ${ }^{3}$ concluded that the actual incident rate of bath-related cardiac arrest in Japan is approximately eight times higher than that in the United States.

Koreans using the public bath (PB) tend to have a similar bathing style of head or neck out-of-water immersion, sitting in hot water. In South Korea, public spas (Jjimjilbangs) made by combining traditional PBs, sauna, and resting space are recently gaining popularity. In South Korea, bath-related cardiac arrests are likely to increase due to the high popularity of Jjimjilbangs and the recent rapid increase in the elderly population. No investigations of bath-related out-of-hospital cardiac arrest (OHCA) have been conducted in South Korea, except for one retrospective study, which described the cause and characteristics of bath-related deaths at autopsy. ${ }^{12}$ Although some previous studies have demonstrated that the outcomes of bath-related OHCA patients were poor, few compared the outcomes of cardiac arrest to other locations. $^{4,5}$

Recently, a Smart Advanced Life Support (SALS) pilot project was introduced in some areas in South Korea, where in the event of a cardiac arrest, the nearest two units of emergency medical services (EMS) are dispatched, and under the directives of an emergency physician, the paramedics could use a manual defibrillator, administer drugs, and perform advanced life support. ${ }^{13}$ Therefore, this study aimed to determine the characteristics and outcomes of PB-related cardiac arrest in South Korea using the SALS registry.

\section{METHODS}

\section{Study design and study setting}

This study was a retrospective observational analysis of data collected from the SALS database between October 2015 and December 2018. In total, nine academic tertiary hospitals and 19 fire stations in eight areas in South Korea participated in this study. The total study area was $7,129 \mathrm{~km}^{2}$ with a population of about 12 million in 2017. We included data on all non-trauma cardiac arrest patients for whom an EMS was dispatched and attended. OHCA patients aged 18 years or younger were excluded from this study. Patients with obvious irreversible death sign, cardiopulmonary resuscitation (CPR) refusal, do-not-resuscitate status, non-cardiac origin arrest, pregnancy, missing or incomplete medical record data, and arrests in ambulance were also excluded. The present study protocol was reviewed and approved by the institutional review board of Ajou University College of Medicine and was waived the requirement of informed consent.

\section{The Korean EMS system}

The Korean EMS system is operated directly by the government. A basic-to-intermediate service level of ambulance is operated in 16 provincial headquarters of the national fire departments, covering a population of approximately 50 millions. ${ }^{14}$ The Korean EMS has recently established a centrally-based, two-tiered system for OHCA patients in which an ambulance is dispatched with 
two or three emergency medical technicians (EMTs), including at least one level 1 EMT who has pre-hospital emergency care skill comparable to EMT-I (intermediate EMT) level in the US. Level 1 EMTs are authorized to administer intravenous fluids, to place endotracheal intubations, and insert laryngeal mask airways for OHCA patients under direct medical oversights. EMTs dispatched to the field perform the conventional basic life support (BLS), then transfer the patient to the nearest hospital. Trained level 1 EMTs are allowed to use medications for advanced cardiac life support (e.g., epinephrine, amiodarone) under direct medical control in SALS pilot project areas. When EMTs arrive on the scene, they perform BLS immediately, obtain the family's consent and conduct SALS if the family agrees. SALS is an advanced field resuscitation by an EMT with video communication-based direct medical oversight. Further detailed explanation of SALS is described in our previous study. ${ }^{13}$ If the family disagrees with SALS, EMTs perform resuscitation just as usual, then transfer to the nearest hospital. EMTs cannot declare death or stop CPR on the scene or during transport based on self-judgement. All patients with $\mathrm{OHCA}$ are transported to the emergency departments unless a physician instructs them to stop CPR by giving a direct medical instruction. ${ }^{15}$

\section{Data source}

After the resuscitation, the EMT fills out the patients' ambulance run sheets that are electronically stored in each provincial EMS headquarter. If SALS was performed, the directing physician completes an oversight physician report, in addition. A trained data manager visits the hospitals to which patients were transported to review the medical records and complete the record according to the Utstein style and construct the SALS database. ${ }^{16}$ Physicians responsible for the database quality control in this study contacted the hospital treating the patient to verify information on outcomes (survival and neurological outcomes) at discharge. Judgment of the patient's outcome was done by the treating physicians based on the Cerebral Performance Category score. CPC scores of 1 (good performance) and 2 (moderate disability) were defined as favorable neurological outcome while a score of 3 (severe cerebral disability), 4 (vegetative state), or 5 (death) were defined as unfavorable neurologic outcomes.

The address at the scene was automatically stored in the EMS headquarters' database, and printed on the ambulance run sheet at the time of dispatch. The location of cardiac arrest was extracted from the address; the details of the place of arrest were obtained from the written records in the ambulance run sheets.

\section{Study outcome}

The primary outcome of this study was survival to discharge, while the secondary outcomes were prehospital return of spontaneous circulation (ROSC) and favorable neurological outcomes that were measured at discharge.

\section{Statistical analysis}

Statistical analysis was performed using IBM SPSS Statistics ver. 21.0 (IBM Corp., Armonk, NY, USA). Nominal variables are expressed as counts and percentages. Continuous variables are expressed as mean and standard deviation. Chi-square test (Fisher exact test) and Student t-test (for continuous variables) at a significance level of $\mathrm{P}<0.05$ were used to test for differences in observed characteristics and outcomes of patients. Using logistic regression analysis including potential confounders (sex, age, witnessed, bystander CPR, bystander automated electrical defibrillator use, shockable cardiac rhythm, and EMS response time), we assessed the odds ratios (ORs) and 95\% confidence intervals (Cls) for survival to discharge.

\section{RESULTS}

\section{Patient flow}

A total of 38,995 cardiac arrest patients were enrolled in the SALS registry during the study period, while 11,889 were included in the final analysis (Fig. 1). A total of 27,106 patients, including 12,363 patients with non-cardiac origin arrest $(12,363)$, obvious irreversible death $(7,652)$, CPR refusal $(3,820)$, aborted CPR $(1,344)$, arrest in ambulance (939), age under 18 years or pregnant (421), do-not-resuscitate (359), and incomplete data (208) were excluded from the final analysis. Of those patients who met the inclusion criteria, $263(2.2 \%)$ cardiac arrests occurred in the PB (PB group) while 11,626 (97.8\%) occurred in other places (non-PB group). Among the PB cardiac arrest cases, 141 (53.6\%) were SALS cases.

Table 1 displays the patient characteristics including EMS factors, time interval, hospital care, and outcome and differences between the two groups. Among the PB group, percentages of male sex and bystander CPR appeared higher than in the non-PB group. Rate of witnessed arrest, shockable rhythm, number of underlying diseases, implementation of SALS, and defibrillation attempt in the PB group were lower than in the non-PB group. EMS time interval including response time and call for EMS life support in the PB group were also shorter than in those of the non-PB group. Overall outcome appeared relatively poor in the PB group for prehospital ROSC (11.4\% vs. $19.5 \%, \mathrm{P}=0.001)$, admission to hospital (7.2\% vs. $18.7 \%, P=0.001)$, survival to hospi- 


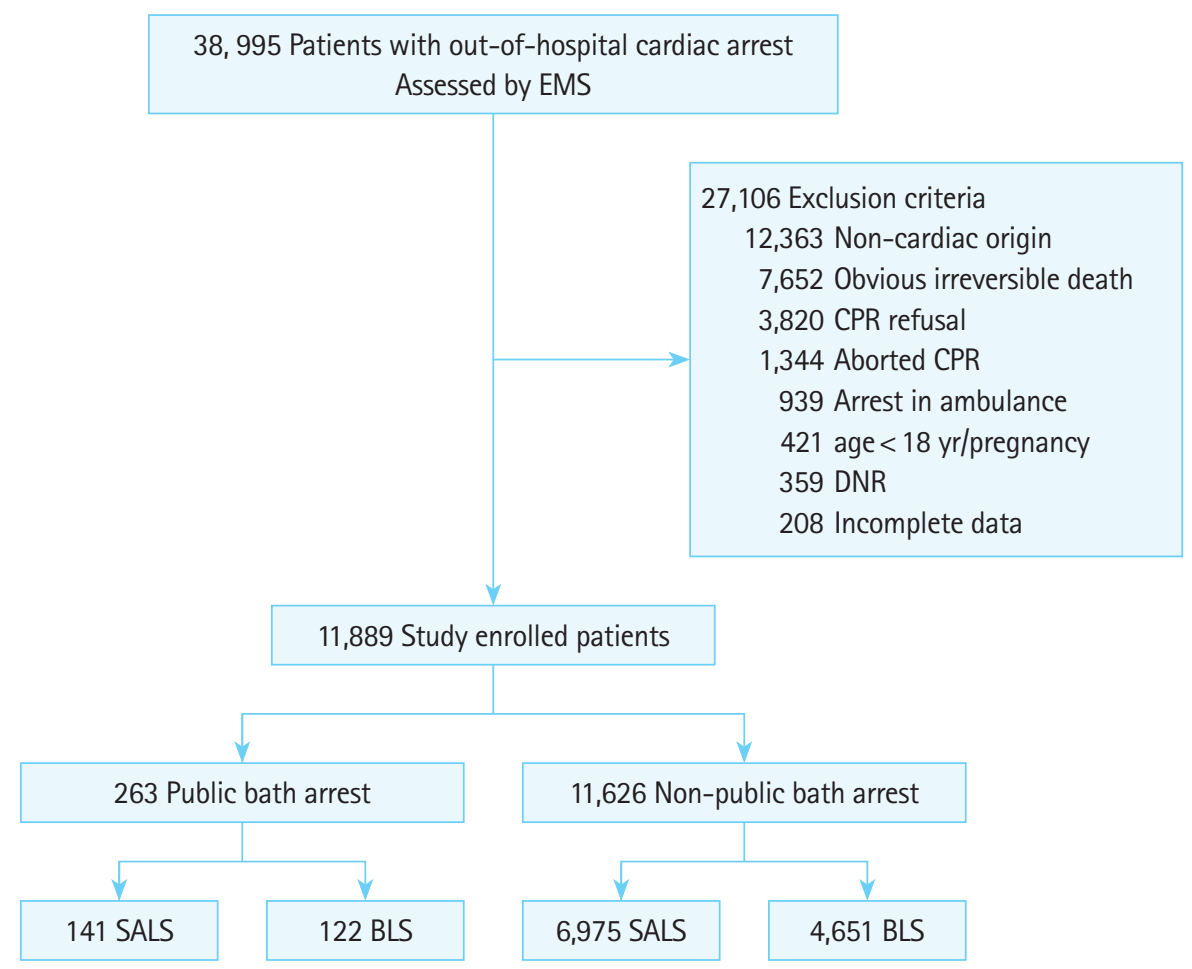

Fig. 1. Flow chart of the included patients. EMS, emergency medical service; CPR, cardiopulmonary resuscitation; DNR, do-not-resuscitate; SALS, Smart Advanced Life Support; BLS, basic life support.

tal discharge $(2.3 \%$ vs. $9.9 \%, \mathrm{P}<0.001)$, and good neurologic recovery (1.9\% vs. $5.8, P=0.007)$.

\section{Distribution of the PB-related OHCA occurrences by month, age, and sex}

Fig. 2 shows the monthly distribution of the number of PB-related OHCA occurrences. Overall, 172 (67.0\%) PB-related OHCAs occurred in the bathtubs. OHCA related to PB occurred mainly in autumn and winter (September to February, $n=167$ ), and the incidence decreased in spring and summer (March to August, $\mathrm{n}=88$ ). As shown in Fig. 3, the individuals' ages ranging from 70 to 79 years showed the highest incidence (38.0\%), followed by ages 80 to $89(29.3 \%)$. Ages ranging from 19 to 49 years were generally with low incidence.

\section{Outcomes of PB- and non-PB-related OHCA patients of SALS and BLS}

In the PB-related arrest patients, prehospital ROSC (17.7\% vs. 4.1\%, $P<0.001)$ and any ROSC $(20.6 \%$ vs. 9.8\%, $P=0.017)$ of performed SALS were significantly higher than that of BLS (Table 2). Hospital admission ( $8.5 \%$ vs. $5.7 \%, \mathrm{P}=0.386)$, survival to discharge rate (2.8\% vs. $1.6 \%, P=0.517)$, and favorable neurologic recovery rate ( $2.1 \%$ vs. $1.6 \%, P=0.772)$ were higher in the SALS group than in the BLS group, but there was no significant difference between the two groups. However, in the non-PB cardiac arrest cases, all outcomes measured improved significantly when SALS was performed.

\section{ORs for prehospital ROSC in PB-related OHCA patients}

Table 3 reveals the OR for prehospital ROSC in PB-related cardiac arrest patients. The OR for prehospital ROSC was $5.87(95 \% \mathrm{Cl}$ 2.01-17.13) when SALS was performed. When age increased by 1 year, prehospital ROSC decreased significantly (OR, 0.96; 95\% Cl, 0.93-0.99). Shockable rhythm was also associated with increased odds of prehospital ROSC $(\mathrm{OR}, 3.58 ; 95 \% \mathrm{Cl}, 1.07-11.98)$. Whereas the $\mathrm{OR}$ for survival to discharge and favorable neurologic recovery were not obtained due to the small number of surviving patients.

\section{The result of the logistic regression analysis for} predicting survival to discharge in OHCA patients

Table 4 displays the results of the multivariate logistic regression analysis for predicting survival to discharge in OHCA patients. The OR for survival to discharge was $0.33(95 \% \mathrm{Cl}, 0.14-0.76)$ when cardiac arrest occurred in the PB. As age and EMS response time increased, the outcome of survival to discharge worsened. Witnessed arrest, bystander CPR, and shockable initial rhythm also were associated with improved outcome of survival to discharge in the OHCA patients. 
Yung Jae Yoo, et al.

Table 1. Baseline characteristics of the study patients

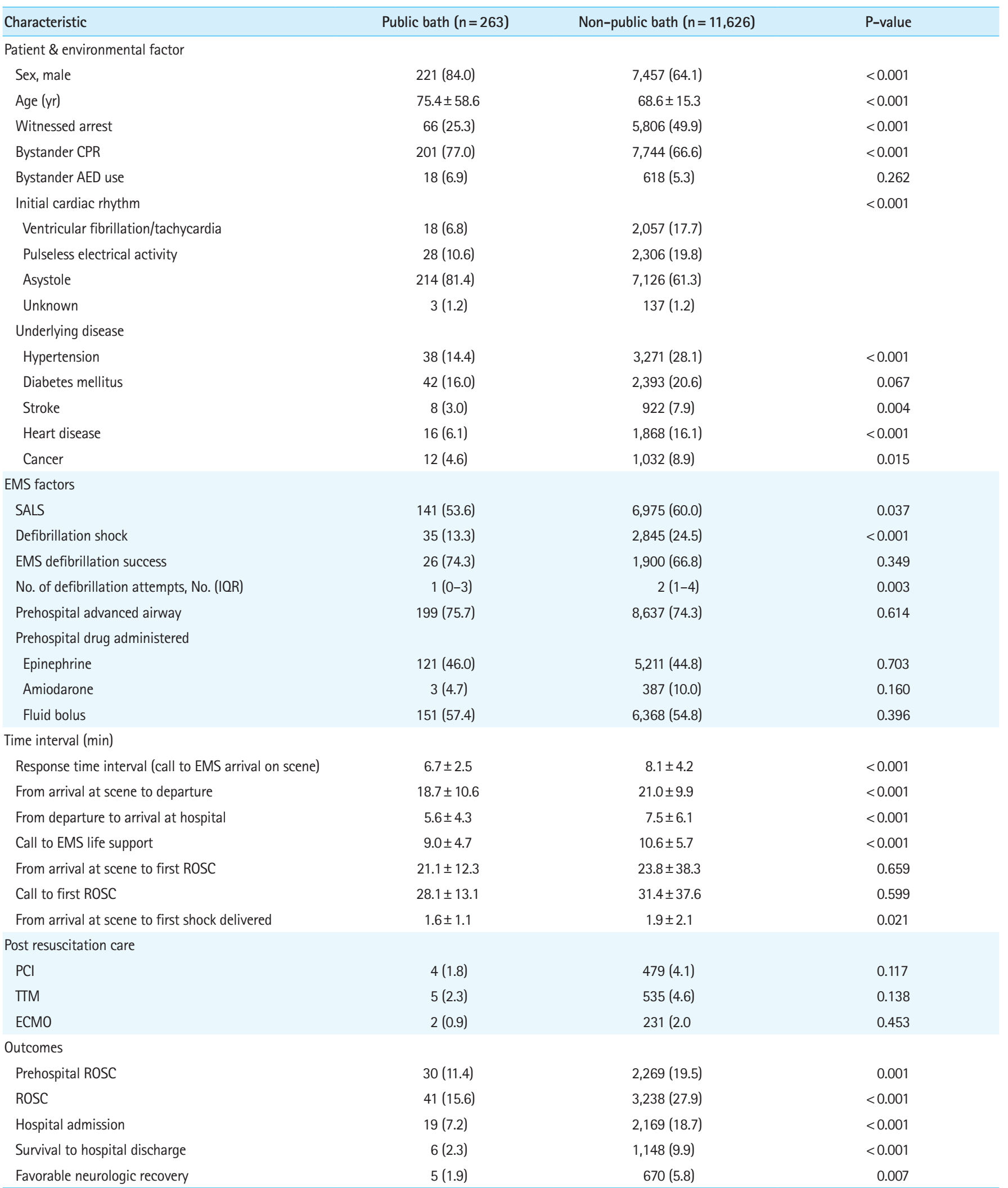

Values are presented as number (\%), mean \pm standard deviation, or median (interquartile range).

CPR, cardiopulmonary resuscitation; AED, automated electrical defibrillator; EMS, emergency medical service; SALS, Smart Advanced Life Support; ROSC, return of spontaneous circulation; $\mathrm{PCl}$, percutaneous coronary intervention; $\mathrm{TM}$, target temperature management; $\mathrm{ECMO}$, extracorporeal membrane oxygenation. 


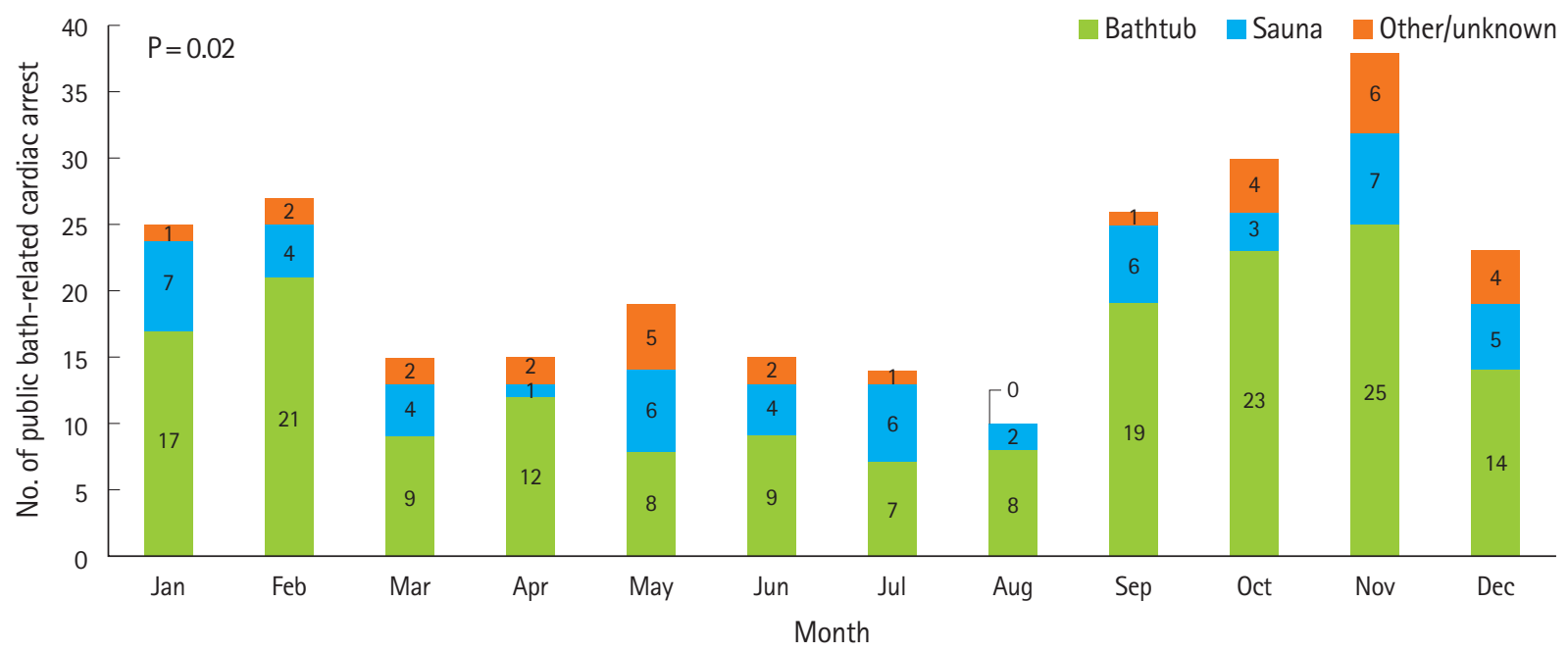

Fig. 2. Distribution of the number of public bath-related cardiac arrests by the month.

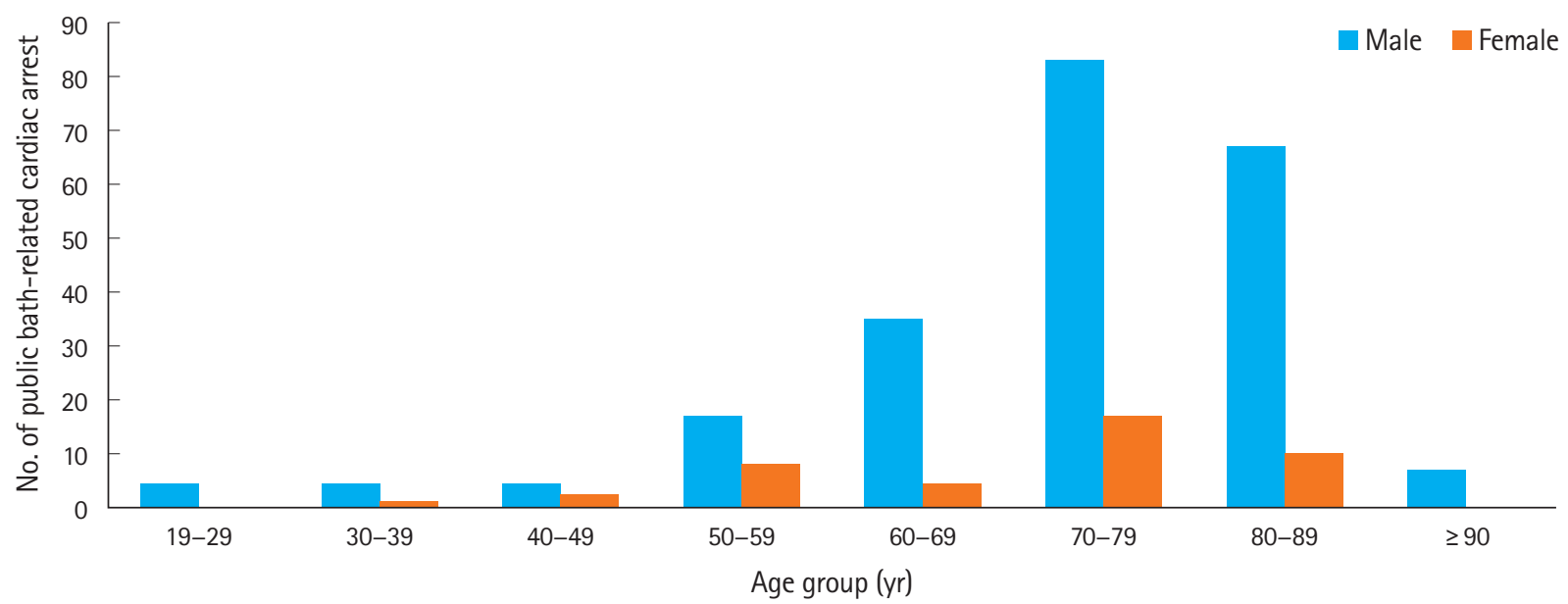

Fig. 3. Distribution of age and sex of public bath-related cardiac arrests.

Table 2. Resuscitation outcomes according to SALS and BLS implementation

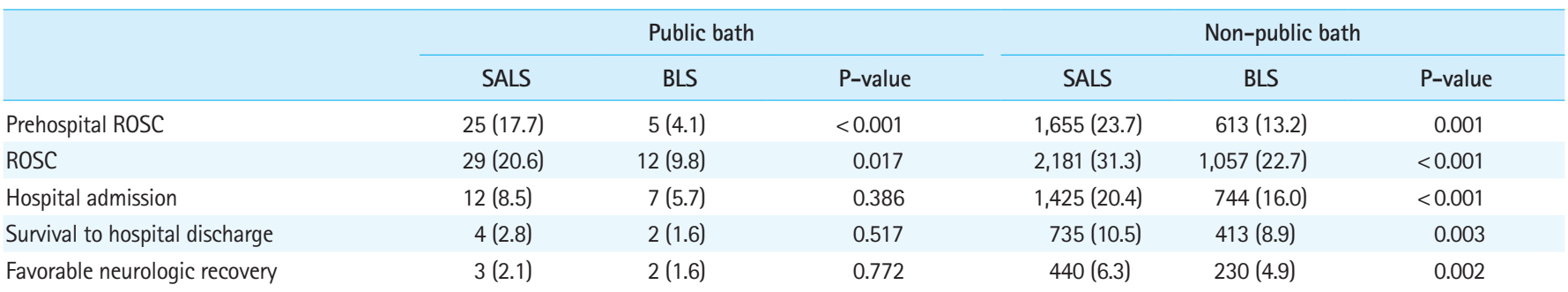

Values are presented as number (\%).

SALS, Smart Advanced Life Support; BLS, basic life support; ROSC, return of spontaneous circulation.

\section{DISCUSSION}

Almost all studies on public bath-related cardiac arrest were conducted in Japan and described the epidemiologic circumstances and pathophysiologic findings based on autopsy findings. ${ }^{3,46-9}$ To the best of our knowledge, this study is the first to evaluate the relationship between cardiac arrest occurrence in the PB and its outcomes directly. We found that the occurrence of OHCA in the PB was associated with poorer outcome. Bath-related cardiac arrests were more likely to increase owing to the high popularity of 
Table 3. ORs for prehospital return of spontaneous circulation in public bath-related out-of-hospital cardiac arrest patients

\begin{tabular}{lcc}
\hline & OR $(95 \% \mathrm{Cl})$ & P-value \\
\hline SALS & $5.87(2.01-17.13)$ & 0.001 \\
Witnessed arrest & $1.84(0.74-4.58)$ & 0.192 \\
Bystander CPR & $1.00(0.35-2.84)$ & 0.999 \\
Bystander AED attached & $0.33(0.04-2.84)$ & 0.327 \\
Shockable rhythm & $3.58(1.07-11.98)$ & 0.039 \\
Age & $0.96(0.93-0.99)$ & 0.004 \\
Sex, male & $0.48(0.18-1.27)$ & 0.142 \\
Response time interval & $1.01(0.86-1.20)$ & 0.876
\end{tabular}

$\mathrm{OR}$, odd ratio; $\mathrm{Cl}$, confidence interval; SALS, Smart Advanced Life Support; CPR, cardiopulmonary resuscitation; AED, automated electrical defibrillator.

PB and spas (Jjimjilbang) as well as the recent rapidly growing elderly population. ${ }^{12}$ Therefore, our study could enable the development of preventive strategies and management guidelines in PBrelated cardiac arrest.

In our study, several characteristics differed between PB-related cardiac arrest patients and the non-PB cardiac arrest patients. Sauna bathing used sensibly and with caution is generally considered to be safe for most healthy people and even among patients with stable cardiovascular disease. ${ }^{2}$ However, a previous study revealed that sudden death in the bathroom was associated with the winter season (56.2\%) and hypertension (37.3\%). ${ }^{5}$ Because almost all cardiac arrests in this previous study occurred in the bathroom at home (84.9\%), it is difficult to compare their result with ours. Underlying medical history like hypertension in PB-related cardiac arrest patients was lower than in the non-PB related cardiac arrest patients. The reason for this difference between both studies may be due to the fact that people's usage tendencies vary depending on the type of baths. ${ }^{2,6}$ All PBs in South Korea have long ago posted warnings that patients with cardiovascular disease such as hypertension should not enter the sauna and warm bathtub. Most people using PBs are aware of this caution and it might have contributed to some selection bias. The majority of PB-related OHCAs occurred during winter, similar to the results of previous studies. ${ }^{3-7,10}$

Increased age was related to the incidence of cardiac arrest occurrence in the PB and to poor prognosis. Our results indicated similar age distributions between Korean and Japanese PB cardiac arrest patients, with most patients being people older than 65 years. ${ }^{3,5,6}$ A recently published autopsy report of bathtub drownings in South Korea revealed that the victims' mean age \pm standard deviation was $61.3 \pm 16.0$ years, which is much lower than our results showed. As the authors mentioned in their report, the autopsy rate in South Korea is extremely low, and autopsy is performed only when necessary. ${ }^{12}$
Table 4. Results of the logistic regression analysis for predicting survival to discharge in out-of-hospital cardiac arrest

\begin{tabular}{lcr}
\hline & OR (95\% Cl) & P-value \\
\hline Occurred in the public bath & $0.33(0.14-0.76)$ & 0.009 \\
Age (per year older) & $0.97(0.97-0.98)$ & $<0.001$ \\
Witnessed arrest & $2.10(1.81-2.43)$ & $<0.001$ \\
Bystander CPR & $1.27(1.08-1.48)$ & 0.008 \\
Shockable rhythm & $5.72(4.97-6.59)$ & $<0.001$ \\
EMS response time interval (per min longer) & $0.94(0.92-0.96)$ & $<0.001$ \\
Bystander AED use & $1.19(0.91-1.56)$ & 0.216 \\
Sex, male & $1.05(0.90-1.23)$ & 0.551
\end{tabular}

$\mathrm{OR}$, odd ratio; $\mathrm{Cl}$, confidence interval; $\mathrm{CPR}$, cardiopulmonary resuscitation; EMS, emergency medical service; $A E D$, automated electrical defibrillator.

Our study revealed that the rate of bystander witnessed cardiac arrest in PBs was very low despite being in a public place. A previous study indicated that the bath-related OHCAs were less likely to be witnessed by bystanders (6.4\% in home baths and $17.6 \%$ in PBs). ${ }^{4}$ Another study also reported that witnessed arrest among bathing patients were more infrequent that that among patients performing other activities. ${ }^{5}$ PBs are basically considered public places, but are also private spaces. Many Koreans do not look at each other because they are completely naked in the PB. When people are in the sauna or Jjimjilbang, sleeping or sitting back in a chair with closed eyes is common; thus, nobody pays attention to the other. This behavioral tendency of these people may delay the detection of cardiac arrest. In addition, since the public spas such as Jjimjilbangs and baths are open 24 hours a day, the likelihood of someone witnessing a cardiac arrest during the night, is likely to decrease.

PB cardiac arrest patients had fewer underlying diseases, faster response time of the EMT, and received more bystander CPR. Nevertheless, the patients in the PB group had lower ROSC, survival to discharge rate, and favorable neurological recovery rate than non-PB patients. As the stay-time increased on the scene to perform SALS, implementation of SALS was associated with increasing prehospital ROSC rates in patients with OHCA and shortened time to ROSC. Time to ROSC remains a significant prognostic factor in patients resuscitated following OHCA, with regard to the risk of death and of neurological outcome. ${ }^{17}$ The implementation of SALS showed improvement of ROSC, but it did not affect survival to discharge rate and favorable neurologic outcome.

One plausible explanation for the poor outcomes could be that the bath-related OHCAs were less likely to have been witnessed by bystanders. As previously mentioned, the characteristics of PBrelated patients were associated with the late recognition of cardiac arrest by bystanders. The late recognition of cardiac arrest 
affected the time to delivery of the first chest compression and poor outcome. It is presumed that the low witness rate may be related to the low rate of shockable rhythm. The low shockable rhythm rate was also associated with the poor prognosis. Other causes for poor outcome could be older age and body temperature of the victims. A previous report suggests that the uniqueness of bath-related OHCAs could be the higher body temperature immediately after cardiac arrest. ${ }^{12}$ In our study, almost all the patients suffering from cardiac arrest were found in warm baths and the saunas. Hyperthermia is associated with an unfavorable neurologic recovery after cardiac arrest. ${ }^{18}$ Furthermore, due to the late recognition, the patients that suffered the cardiac arrest would have been exposed to a higher temperature environment for a longer period. Therefore, our study showed that another strategy should be to provide advanced life support to PBrelated OHCA patients.

Considering that the survival to discharge rate and the neurological recovery rate of cardiac arrest were lower for PB-related than non-PB-related cardiac arrest, the prevention of PB-related cardiac arrest is important. The Japan Resuscitation Council Resuscitation Guidelines 2015 already provides some preventive instructions specific to bath-related OHCAs. ${ }^{19}$ For earlier recognition of cardiac arrest by bystanders, there is need for more attempts to inform the public of the risk of sudden cardiac arrest during bathing and the countermeasures, including a notice. Installing electronic devices using gestures, and motion-tracking recognition technology in saunas or bathtubs could be other favorable options for earlier detection of cardiac arrest. In addition, the employees of PB facilities should be required to complete a BLS training course, including early recognition of cardiac arrest, CPR implementation, and automated electrical defibrillator use.

This study had several limitations. First, our data were retrospectively collected, the cause of the cardiac arrest could not be clarified, and likewise, no blood alcohol concentration was obtained. ' Secondly, 1- or 6-month outcomes after cardiac arrest were not included in the analysis. Thirdly, it was difficult to select a control group to compare with the PB group. We estimated that the comparison of PB and other places where OHCA occur would also not be appropriate for the purpose of this study. Further investigation including prevention and flexible response strategies for PB-related OHCA patients is needed.

In conclusion, the characteristics of PB-related OHCA differed from those of the other OHCA. All outcomes of PB-related OHCA were poorer than for non-PB-related OHCA. Further treatment strategy should be developed to improve the outcome in PB-related cardiac arrest.

\section{CONFLICT OF INTEREST}

No potential conflict of interest relevant to this article was reported.

\section{ACKNOWLEDGMENTS}

This study was supported by Ministry of Health and Welfare (090-091-2800-2832-309) and a grant funded by the Soonchunhyang University, Collage of Medicine (20180012).

We truly thank Dr. Han Deok Youn for his support to help complete this study. He, the director of National Emergency Medical Center, was a pioneer in building disaster response system and emergency medical system of South Korea. Our deepest sympathies go out to him and his family. May God give him the peace that he was seeking.

\section{REFERENCES}

1. Valtakari P. The sauna and bathing in different countries. Ann Clin Res 1988;20:230-5.

2. Laukkanen JA, Kunutsor SK. Is sauna bathing protective of sudden cardiac death?: a review of the evidence. Prog Cardiovasc Dis 2019;62:288-93.

3. Satoh F, Osawa M, Hasegawa I, Seto Y, Tsuboi A. "Dead in hot bathtub" phenomenon: accidental drowning or natural disease? Am J Forensic Med Pathol 2013;34:164-8.

4. Kiyohara K, Nishiyama C, Hayashida S, et al. Characteristics and outcomes of bath-related out-of-hospital cardiac arrest in Japan. Circ J 2016;80:1564-70.

5. Nishiyama C, Iwami T, Nichol G, et al. Association of out-ofhospital cardiac arrest with prior activity and ambient temperature. Resuscitation 2011;82:1008-12.

6. Hayashi T, Ago K, Ago M, Ogata M. Bath-related deaths in Kagoshima, the southwest part of Japan. Med Sci Law 2010;50: $11-4$.

7. Suzuki H, Hikiji W, Tanifuji T, Abe N, Fukunaga T. Characteristics of sudden bath-related death investigated by medical examiners in Tokyo, Japan. J Epidemiol 2015;25:126-32.

8. Suzuki H, Tanifuji T, Abe N, Shibata M, Hikiji W, Fukunaga T. Epidemiology of alcohol-related death while bathing in a bathtub. Nihon Arukoru Yakubutsu Igakkai Zasshi 2015;50:59-65.

9. Suzuki M, Shimbo T, Ikaga T, Hori S. Sudden death phenomenon while bathing in Japan: mortality data. Circ J 2017;81: 1144-9.

10. Chiba T, Yamauchi M, Nishida N, Kaneko T, Yoshizaki K, Yoshioka N. Risk factors of sudden death in the Japanese hot bath 
in the senior population. Forensic Sci Int 2005;149:151-8.

11. Traphagan JW. Culture and long-term care: the bath as social service in Japan. Care Manag J 2004;5:53-60.

12. Yang $\mathrm{K}$, Choi BH, Lee $B$, Yoo SH. Bath-related deaths in Korea between 2008-2015. J Korean Med Sci 2018;33:e108.

13. Kim C, Choi HJ, Moon H, et al. Prehospital advanced cardiac life support by EMT with a smartphone-based direct medical control for nursing home cardiac arrest. Am J Emerg Med 2019;37: 585-9.

14. Kang K, Kim T, Ro YS, Kim YJ, Song KJ, Shin SD. Prehospital endotracheal intubation and survival after out-of-hospital cardiac arrest: results from the Korean nationwide registry. Am J Emerg Med 2016;34:128-32.

15. Ro YS, Shin SD, Song KJ, et al. A trend in epidemiology and outcomes of out-of-hospital cardiac arrest by urbanization level: a nationwide observational study from 2006 to 2010 in South Korea. Resuscitation 2013;84:547-57.

16. Cummins RO, Chamberlain DA, Abramson NS, et al. Recommended guidelines for uniform reporting of data from out-of- hospital cardiac arrest: the Utstein Style. Task Force of the American Heart Association, the European Resuscitation Council, the Heart and Stroke Foundation of Canada, and the Australian Resuscitation Council. Ann Emerg Med 1991;20:861-74.

17. Kjaergaard J, Nielsen $N$, Winther-Jensen $M$, et al. Impact of time to return of spontaneous circulation on neuroprotective effect of targeted temperature management at 33 or 36 degrees in comatose survivors of out-of hospital cardiac arrest. Resuscitation 2015;96:310-6.

18. Zeiner A, Holzer M, Sterz F, et al. Hyperthermia after cardiac arrest is associated with an unfavorable neurologic outcome. Arch Intern Med 2001;161:2007-12.

19. Japan Resuscitation Council. The Japan Resuscitation Council resuscitation guidelines 2015: education, implementation, and teams [Internet]. Tokyo: Japan Resuscitation Council [cited 2019 Jul 1]. Available from: https://www.japanresuscitationcouncil. org/wp-content/uploads/2016/04/b7b5b647189bc07f38f6fecf014cf5d9.pdf. 